\title{
Tratamiento laparoscópico de la hernia paraesofágica y la utilización de mallas
}

\author{
Laparoscopic treatment of paraesophageal hernia and the use of meshes
}

\author{
Jorge Fernando Ugalde Velásquez,* Eduardo Torices Escalante, ${ }^{* *}$ \\ Horacio Olvera Hernández, ${ }^{* * *}$ Francisco Javier Shiordia Puente****
}

\begin{abstract}
Palabras clave:
Hernia hiatal, hernia paraesofágica, hernia hiatal gigante, materiales protésicos,

funduplicatura de

Nissen, tratamiento

laparoscópico.

Key words: Hiatal hernia, paraesophageal hernia, giant hiatal hernia, prosthetic materials, Nissen fundoplication, laparoscopic treatment.
\end{abstract}

\footnotetext{
* Servicio de Cirugía General y Laparoscópica. Hospital Regional $1^{\circ}$ de Octubre ISSSTE, Ciudad de México.

** Servicio de Cirugía General y Laparoscópica. Jefe del Servicio de Endoscopia. ISSSTE. *** Jefe del Servicio de Cirugía Laparoscópica. Hospital Regional $1^{\circ} \mathrm{de}$ Octubre ISSSTE, Ciudad de México.

**** Servicio de Cirugía General, Hospital

Ángeles Roma.
}

Recibido: 08/09/2016 Aceptado: 12/07/2017

\section{RESUMEN}

Introducción: La hernia hiatal paraesofágica representa del 5 al $10 \%$ de las hernias de hiato que se operan. Su importancia radica en el riesgo de aparición de complicaciones graves. Actualmente, la reparación laparoscópica es el procedimiento de elección. Se ha observado una disminución en la recidiva con el uso de materiales protésicos. Objetivos: Evaluar el resultado del manejo laparoscópico con distintos tipos de mallas analizando la calidad de vida, evolución postoperatoria, recidiva y presencia de complicaciones en pacientes con hernia paraesofágica. Material y métodos: Se realizó una revisión de la evolución de 19 pacientes intervenidos por vía laparoscópica de 2008 a 2015. Todos fueron documentados con panendoscopia, tomografía computada, $\mathrm{pH}$-metría y manometría. Se les dio seguimiento a intervalos de tres, seis y 12 meses después de la cirugía. Se realizó un estudio de trago de bario a los tres y 12 meses después de la cirugía y una panendoscopia a los seis meses. Todos los pacientes tienen tomografía de control anualmente. Resultados: Fueron intervenidos 15 pacientes masculinos y cuatro femeninos, con edades de 40 a 78 años; tiempo quirúrgico promedio, 120 minutos; estancia hospitalaria promedio, cuatro días; no se reportaron complicaciones trans- ni postoperatorias; no hubo conversión a cirugía abierta. El reporte de mejoría clínica mostró resultados excelentes y buenos en 15 pacientes, tres permanecen con disfagia moderada y uno con disfagia leve. Hasta la fecha, a nueve años del primer paciente, no ha habido ninguna complicación relacionada con las mallas utilizadas. No se ha presentado recidiva de la hernia en ninguno. Conclusiones: La alta recidiva de la hernia paraesofágica ha generado un incremento en el uso de materiales protésicos para reforzar el cierre. La mallas bicapa y biológicas son seguras y muestran una baja tasa de complicaciones postoperatorias. Consideramos que su uso debe estar basado en la evidencia de la literatura y en la experiencia de cada grupo quirúrgico.

\section{ABSTRACT}

Introduction: Paraesophageal hiatal hernia represents $5-10 \%$ of the hiatal hernias that are operated. Its importance lies in the risk of serious complications. Currently laparoscopic repair is the procedure of choice. We have observed a decrease in recurrence with the use of prosthetic materials. Objectives: To assess the outcome of laparoscopic management with different types of mesh analyzing the quality of life, postoperative course, recurrence and the presence of complications in patients with paraesophageal hernia. Material and methods: A review of the evolution of 19 patients who underwent laparoscopic intervention from 2008 to 2015 was performed. All were documented by panendoscopy, computed tomography, $\mathrm{pH}$-metry and manometry. They were monitored at intervals of three, six and 12 months after surgery. A study of barium swallow was performed at three and 12 months after surgery, and upper endoscopy at six months. All patients have a control scan annually. Results: Fifteen male and four female patients were operated on, aged 40-78 years; the mean operative time was 120 minutes; the average hospital stay was four days; no trans- or postoperative complications were reported; there was no conversion to open surgery. The report of clinical improvement showed excellent and good results in 15 patients; three remain with moderate dysphagia and one with mild dysphagia. Today, nine years after the first patient, there have been no complications related to the mesh used. There has been no recurrence of the hernia in any case. Conclusions: The high recurrence of paraesophageal hernia has generated an increase in the use of prosthetic materials to reinforce the closure. Bilayer and biological meshes are safe and show a low rate of postoperative complications. We believe that their use should be based on evidence from the literature and the experience of each surgical group. 


\section{INTRODUCCIÓN}

L a hernia hiatal es una condición adquirida - que constituye uno de los diagnósticos endoscópicos más frecuentemente realizados, aunque en muchos casos es sobrediagnosticada. ${ }^{1,2}$ Clásicamente, la hernia hiatal se ha clasificado en tres tipos, según Hill. Las hernias paraesofágicas son del tipo II; algunos autores han aumentado el grado IV, por ser complejas, en las que gran parte del estómago es intratorácico; pueden incluir otros órganos como colon, bazo, epiplón y/o intestino delgado. ${ }^{1}$

Es una condición muy común, con una frecuencia de 40 a $60 \%$ en la edad adulta. El $95 \%$ son pequeñas hernias deslizantes tipo I y raramente están asociadas a complicaciones serias. El restante $5 \%$ está clasificado como hernias paraesofágicas gigantes y se asocian a complicaciones significativas. ${ }^{3}$

Se ha demostrado que la cirugía antirreflujo en hernias paraesofágicas con abordaje laparoscópico presenta mejores resultados a corto plazo que el resto de las técnicas, ocasionando menos dolor y morbilidad para el paciente, reduciendo los costos de hospitalización y estancia hospitalaria; esto incluye la técnica abierta. ${ }^{4}$ Debido a lo anterior, actualmente la reparación laparoscópica es de elección para el tratamiento de esta patología. 5,6

Ahora bien, con el tiempo, la funduplicatura puede debilitarse y ocasionar síntomas de reflujo; la reincidencia de la hernia se ha descrito en un $1.3 \%$ de los pacientes en las primeras 48 horas posteriores a la cirugía y en un $16-42 \%$ de ellos en periodos más prolongados (cinco años). ${ }^{4,7}$

Para reducir la recurrencia, algunos cirujanos han usado diferentes tipos de mallas para reforzar el cierre del hiato $y$, de esa manera, asegurar que la funduplicatura permanezca en su lugar. ${ }^{8}$

Las complicaciones con el uso de mallas sintéticas son resultado de la fibrosis, como formación de estenosis, erosión esofágica, migración de malla y disfagia tardía a largo plazo, que podrían evitarse. Para tratar de resolver estas situaciones, se han desarrollado mallas de teflón (bicapa) y biológicas: de dermis acelular de cadáver humano (human acellular cadaveric dermis, HACD), de submucosa de intestino delgado porcino (porcine small intestine submucosa, SIS), de colágena dérmica porcina (porcine dermal collagen, PDC) o de pericardio de bovino. ${ }^{9}$

Presentamos una revisión de nuestra experiencia desde 2008 y hacemos una comparación con los reportes obtenidos en la literatura. En este estudio describimos el desenlace de 19 pacientes a quienes se les reparó la hernia hiatal paraesofágica (HHP) gigante por laparoscopia y utilización de malla de polipropileno, dual mesh y biológica SIS.

\section{Objetivos}

- Evaluar los resultados obtenidos en el manejo laparoscópico con mallas de material no absorbible, bicapa y biológicas en pacientes con diagnóstico de hernia paraesofágica.

- Evaluar la asociación del material utilizado en la reparación laparoscópica con las complicaciones, evolución postoperatoria, recidiva y cambios en la calidad de vida.

\section{MATERIAL Y MÉTODOS}

Se realizó una revisión de la evolución de 19 pacientes intervenidos por vía laparoscópica de 2008 a 2015. Se obtuvieron todos los datos demográficos, como sexo, edad, tiempo de evolución de la enfermedad y síntomas, estudios previos, comorbilidades. Todos los pacientes tuvieron evidencia de enfermedad por reflujo gastroesofágico (ERGE) y de HHP. Todos contaban con serie esófago gastroduodenal (SEGD), panendoscopia, pH-metría, manometría, tomografía axial computada (TAC) toracoabdominal simple y contrastada.

Ninguno de los pacientes tenía enfermedad maligna; no habían sido intervenidos de abdomen superior.

Previamente a la cirugía, todos los pacientes fueron entrevistados e interrogados acerca de la presencia de síntomas de reflujo gastroesofágico (ardor retroesternal, regurgitación, dolor precordial, síntomas atípicos de reflujo) y los datos fueron recabados de acuerdo al GORD$\mathrm{HRQL}$, que es un cuestionario de 11 elementos diseñado para realizar una evaluación práctica y confiable de la severidad de los síntomas de 
ERGE y calidad de vida. Cada ítem se mide desde cero (mejor resultado) a cinco (peor resultado), con cada resultado basado en una escala descriptiva.

\section{Técnica quirúrgica}

Las bases de nuestra técnica fueron:

- Utilización de una técnica de cuatro puertos (3-10 mm, 1-5 mm) en todos los pacientes.

- Revisión completa de la cavidad.

- Disección cuidadosa de ambos pilares.

- Identificación del nervio vago anterior.

- Reducción gentil del contenido abdominal del tórax a la cavidad (estómago, colon, intestino delgado, bazo, epiplón).

- Resección, en la medida de lo posible, del saco herniario y/o sus medios de fijación al hiato.

- Evaluación de la longitud del esófago y diagnóstico de esófago corto.

- Realización del cierre de los pilares libre de tensión.

- Colocación y fijación de malla prolene, dual mesh o biológica alrededor del esófago, sobre el diafragma. El uso de éstas dependió de la disposición que se tenía en la institución en el momento de la cirugía.
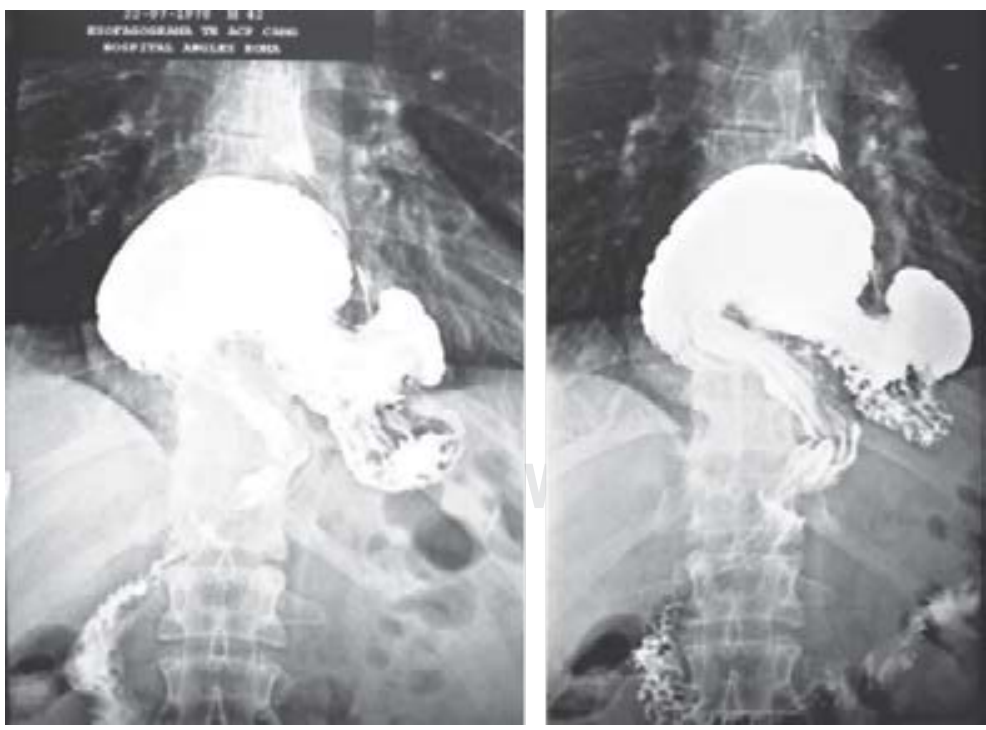

Figura 1. Serie esofagogastroduodenal.
- Especial cuidado en que la distancia de la malla al esófago fuera de un centímetro.

- En caso necesario, reparación del defecto crural utilizando la malla dual mesh no como reforzamiento sino como techo para conseguir un cierre libre de tensión, o en caso de tener un tejido muscular de pobre calidad que impidiera adecuada colocación de suturas.

- Realización de funduplicatura Nissen o Toupet en caso de demostrar una dismotilidad esofágica.

\section{Trago de bario}

En todos los pacientes se realizó en el postoperatorio un examen radiológico esofágico estándar a los tres y 12 meses postquirúrgicos. Con base en lo mencionado por la literatura, definimos como "hernia hiatal paraesofágica gigante" a la migración al tórax de más un tercio del estómago con o sin otros órganos (Figura 1). ${ }^{10,11}$

El estudio fue aprobado por el comité de ética de los diferentes hospitales en donde se realizó la intervención y se condujo de acuerdo con los estándares éticos de la declaración de Helsinki. Todos los pacientes firmaron consentimiento informado.

\section{RESULTADOS}

Fueron intervenidos por vía laparoscópica 19 pacientes: 15 masculinos y cuatro femeninos, entre 40 y 78 años de edad. Se presentan los datos de tiempo de evolución de la HHP, el índice de DeMeester preoperatorio, el tamaño de la hernia en centímetros y el tiempo quirúrgico (Cuadro I), además de las comorbilidades que tenían (Cuadro II).

La presentación clínica preoperatoria tuvo predominio, con ardor retroesternal y síntomas respiratorios (Cuadro III).

En la endoscopia preoperatoria, 52\% de los pacientes tenían esofagitis grado $C$; valores descritos en el cuadro $I V$.

En 13 casos se resecó el saco herniario; en todos se realizó reducción y liberación de los medios de fijación del saco a la cavidad abdominal (Figuras 2 y 3). En 15 casos, cierre de los pilares (Figuras 4 y 5); en los seis casos 


\begin{tabular}{lcccrr}
\multicolumn{5}{c}{ Cuadro I. Estadísticos descriptivos. } \\
& N & Mínimo & Máximo & Media & Desv. típ. \\
\hline & 19 & 40 & 78 & 59.78 & 10.741 \\
\hline Edad (años) & 19 & 1 & 10 & 5.00 & 3.254 \\
Tiempo de evolución de la & & & & & \\
HHP (años) & 19 & 19 & 133 & 86.28 & 31.223 \\
DeMeester (índice) & 19 & 4 & 14 & 8.33 & 3.742 \\
Tamaño de la hernia (cm) & 19 & 90 & 180 & 123.22 & 23.222 \\
Tiempo quirúrgico (min) & 19 & & & & \\
\end{tabular}

\begin{tabular}{lr}
\multicolumn{2}{c}{$\begin{array}{c}\text { Cuadro II. Comorbilidades } \\
\text { agregadas en los pacientes. }\end{array}$} \\
\hline Comorbilidades & $\%$ (n) \\
\hline Hipertensión arterial & $26(5)$ \\
Diabetes mellitus tipo II & $21(4)$ \\
EPOC & $21(4)$ \\
Obesidad & $5(1)$ \\
Síndrome metabólico & $52(10)$ \\
Asma & $16(3)$ \\
\hline
\end{tabular}

Cuadro III. Síntomas preoperatorios.

Síntomas preoperatorios: \% de presentación (n)

\begin{tabular}{lll}
\hline Ardor retroesternal & $63(12)$ \\
Síntomas respiratorios & $57(11)$ \\
Dolor epigástrico & $52(10)$ \\
Disfagia & $42(8)$ \\
Regurgitación & $47(9)$ \\
Dolor torácico & $42(8)$
\end{tabular}

\begin{tabular}{|lcc|}
\hline \multicolumn{3}{|c|}{$\begin{array}{c}\text { Cuadro IV. Clasificación } \\
\text { endoscópica preoperatoria. }\end{array}$} \\
\hline $\begin{array}{l}\text { Clasificación } \\
\text { de los Ángeles }\end{array}$ & $\begin{array}{c}\text { Núm. de } \\
\text { pacientes }\end{array}$ & Porcentaje \\
\hline Grado A & 2 & 10.5 \\
Grado B & 2 & 10.5 \\
Grado C & 10 & 52.6 \\
Grado D & 5 & 26.3 \\
Total & 19 & 100 \\
\hline
\end{tabular}

iniciales se utilizó malla de polipropileno, en 12, colocación de malla bicapa (Figura 6), y en uno, malla biológica alrededor del esófago y fijación al diafragma con puntos o grapas (Figura 7); se finalizó el procedimiento con funduplicatura de Nissen. ${ }^{12,13}$

El seguimiento que se lleva hasta el momento es de nueve años (Figura 8) con el paciente más antiguo y 12 meses con el más reciente; no ha habido ninguna recidiva de la hernia. En los seis casos en los que no resecamos el saco en su integridad, hasta la fecha no se han presentado complicaciones ni recidiva de la hernia.

Los seis primeros pacientes fueron tratados con malla de polipropileno; hasta el momento no se han tenido complicaciones debidas al tipo de material utilizado. En el último paciente incluido en esta serie, en 2015, se utilizó malla biológica.

El tiempo quirúrgico promedio fue de 120 minutos; la estancia hospitalaria promedio fue

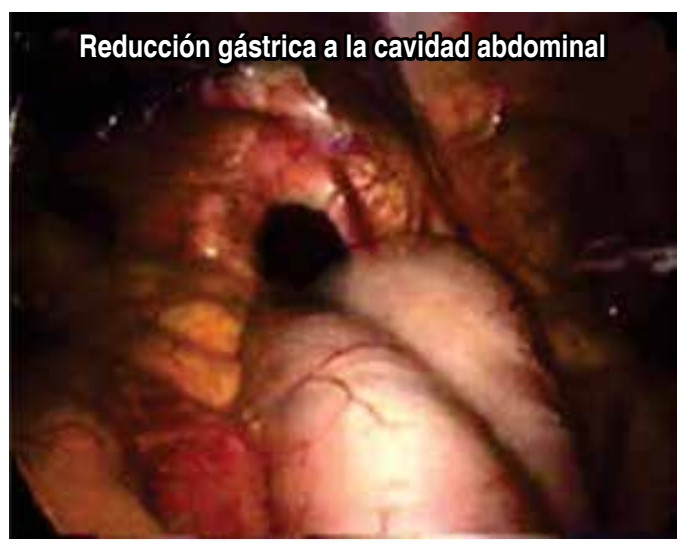

Figura 2. Reducción del contenido herniario.

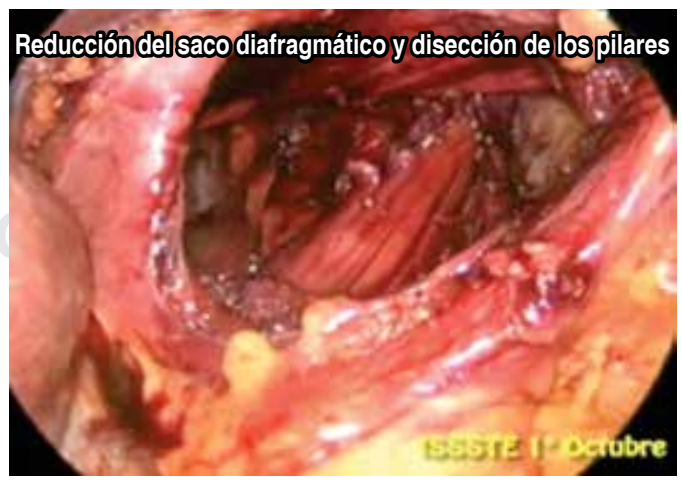

Figura 3. Disección del saco y los pilares. 
de cuatro días, sin reportarse complicaciones transoperatorias; en el postoperatorio, la complicación más común fue neumonía (Cuadro V). Las complicaciones que surgieron en el postoperatorio se resolvieron favorablemente con manejo médico y no ameritaron ningún

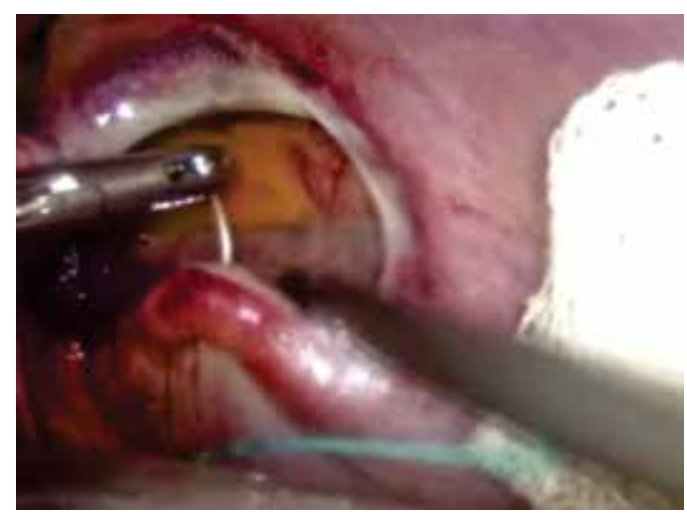

Figura 4. Cierre del defecto diafragmático.

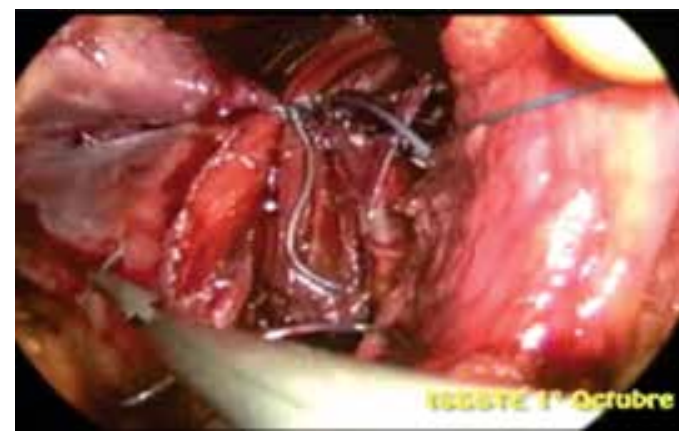

Figura 5. Cierre de los pilares del diafragma (derecha).

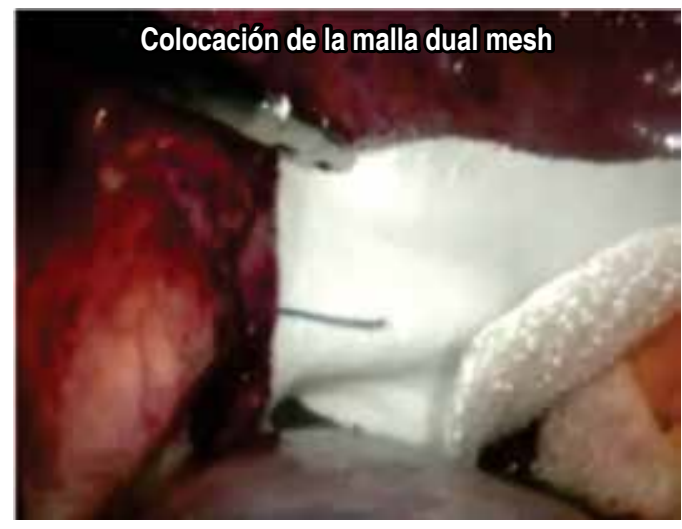

Figura 6. Colocación de la malla bicapa para reforzamiento del cierre.

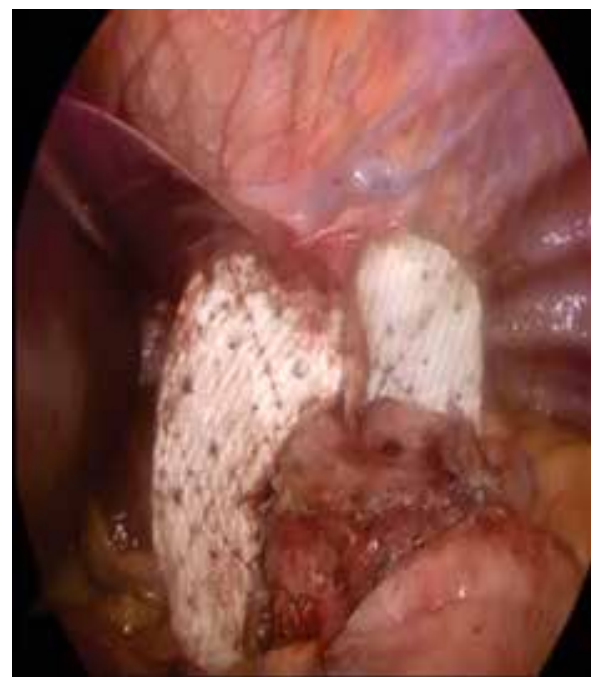

Figura 7. Colocación de la malla SIS para reforzamiento del cierre.

Línea del tiempo en el seguimiento de los pacientes

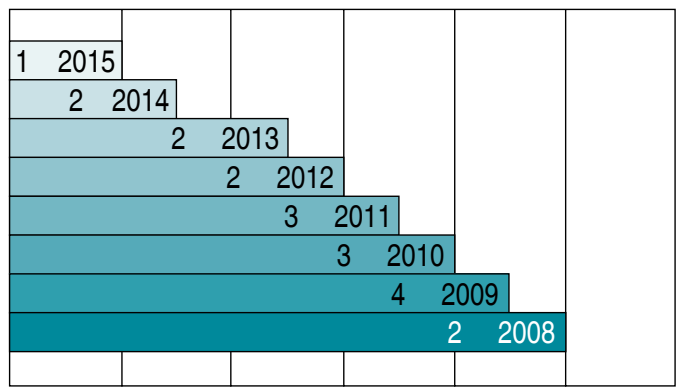

Figura 8. Seguimiento de los pacientes en la línea del tiempo.

Cuadro V. Complicaciones en el postoperatorio inmediato y mediato.

\begin{tabular}{lcc} 
Complicaciones & $\begin{array}{c}\text { Núm. de } \\
\text { pacientes }\end{array}$ & Porcentaje \\
\hline Neumonía & 3 & 15 \\
Atelectasia & 3 & 15 \\
Neumotórax & 1 & 5 \\
Mortalidad & 0 & 0 \\
Infección & 0 & 0 \\
Sangrado mayor al & 0 & 0 \\
permisible & & \\
Recidiva de hernia & 0 & 0 \\
Conversión & 0 & 0
\end{tabular}


procedimiento que prolongara la estancia hospitalaria; no hubo conversión a cirugía abierta en nuestra serie.

En el cuestionario de calidad de vida GORD-HRQL, hay hasta el momento 15 casos con resultados buenos a excelentes, tres casos con disfagia moderada y un caso con disfagia leve. No hemos encontrado complicaciones inherentes al uso de mallas de polipropileno, bicapa ni biológicas en nuestros pacientes.

\section{DISCUSIÓN}

La hernia hiatal paraesofágica representa un $5-10 \%$ de todas las hernias de hiato. Su importancia radica en el riesgo de aparición de complicaciones graves, ${ }^{14}$ por ello se recomienda el tratamiento quirúrgico en todos los casos. ${ }^{15,16} \mathrm{El}$ manejo conservador debe ser cuidadosamente valorado en casos especiales (pacientes seniles, comorbilidades, asintomáticos). ${ }^{17}$

La edad de presentación más frecuente es entre la cuarta y sexta décadas de la vida; no existe diferencia en cuanto al sexo. Cuando su evolución es prolongada, la diferencia de presiones entre tórax y abdomen, la laxitud de la membrana freno-esofágica y de los elementos de fijación gastroesofágicos originan un aumento de volumen herniario que puede acompañarse de deslizamiento gástrico y eventual formación de un vólvulo gástrico mesentérico-axial, con más frecuencia por la fijación del pedículo vascular.

En un diseño presentado por Ballian ${ }^{18}$ para detectar los factores que afectaban la morbimortalidad en una serie de 980 pacientes, observó que si la cirugía era electiva, la mortalidad era del $1.1 \%$, y si era de urgencia, del $8 \%$. Factores adicionales asociados a la mortalidad incluyen: mayores de 65 años, bajo índice de masa corporal, historia de insuficiencia cardiaca, enfermedad vascular cerebral (EVC), demencia, enfermedad pulmonar, úlcera péptica y malignidades en los últimos cinco años. En nuestra serie, la media de la edad fue de casi 60 años; sin embargo, no hay pacientes con historia de insuficiencia cardiaca, EVC, demencia, úlcera péptica o malignidades.

El 56\% de nuestros pacientes tuvieron esofagitis C de los Ángeles, con una media del tamaño de la hernia de $8.3 \mathrm{~cm}$ y un tiempo promedio de evolución antes de la cirugía de cinco años, factores que evidentemente nos hablan de la historia natural de la enfermedad y de los estragos que el tiempo hace con toda la fisiopatología subyacente al padecimiento.

El primer reporte de reparación por laparoscopia de una HHP fue publicado en 1992 por Cuschieri. ${ }^{19}$ Antes de ese momento, todas las cirugías eran abiertas, evidentemente. Cuando comparamos los resultados entre cirugía abierta y cirugía laparoscópica nos remitimos a un estudio prospectivo que reportó en 2011 una comparación entre 2,069 pacientes con HHP intervenidos por laparoscopia y 657 operados de manera abierta; en él se reportó una menor estancia hospitalaria (3.7 versus 8.3 días), un descenso en el requerimiento de terapia intensiva (13 versus $35 \%)$ y un más bajo índice de complicaciones (2.7 versus $8.4 \%$ ), así como de readmisiones a 30 días (1.4 versus $3.4 \%$ ) y menores costos $(\$ 15,227$ USD de la laparoscópica versus $\$ 24,263$ USD de la abierta). La mortalidad fue de $0.4 \%$ para la laparoscopia versus $0 \%$ para los abiertos. ${ }^{20}$ Lo anterior está de acuerdo con nuestros resultados, donde tuvimos una estancia hospitalaria promedio de 3.68 días; ningún paciente requirió terapia intensiva; el porcentaje de complicaciones fue del 5 al $15 \%$, todas ellas pulmonares, principalmente, sin readmisión a los 30 días.

En los últimos años, los cirujanos han empezado a reportar su experiencia con la reparación laparoscópica de grandes hernias hiatales, y este abordaje es ahora considerado la técnica de elección para el tratamiento de la hernia paraesofágica gigante. ${ }^{21,22}$ La laparoscopia ofrece varias ventajas comparada con la técnica abierta, incluyendo visualización del mediastino posterior, manejo preciso de los tejidos, incisiones más pequeñas, con disminución del dolor postoperatorio y recuperación más temprana. A pesar de los buenos resultados que se obtienen con laparoscopia en la reparación de la HHP, una investigación reciente en miembros de la Cardio Thoracic Surgery Network reveló que sólo $48 \%$ de ellos reparan la HHP vía laparoscópica, mientras que $35 \%$ realizan toracotomía y $17 \%$ lo hacen vía laparotomía. ${ }^{23}$

Se ha propuesto el uso rutinario de materiales protésicos para reforzar la hernia en 
caso de ser muy grande; de esta manera se ha reducido la recidiva hasta el $10 \% .{ }^{24-26} \mathrm{Sin}$ embargo, existen varios argumentos contra el uso de prótesis, principalmente por la disfagia a corto y largo plazo, producto de la fibrosis alrededor del esófago y erosión o fistulización por el cuerpo extraño. ${ }^{27-29}$

Algunos de los factores pronósticos de recidiva tras la reparación laparoscópica pueden incluir las características anatómicas del hiato (tamaño del defecto, existencia de tensión, debilidad diafragmática), el tipo de reparación (puntos sueltos, utilización de pledget, etcétera), las maniobras adicionales de fijación (técnica de Toupet, pexia, gastrostomía, etcétera) y las características de los pacientes (trabajo pesado, estreñimiento, tos crónica, etcétera). ${ }^{30}$

Una revisión de la literatura china en 2013 encontró que un reforzamiento con malla bicapa de teflón mejoraba significativamente los síntomas relacionados con la hernia hiatal. ${ }^{31}$ Por otro lado, Zou confirmó que el uso de este tipo de mallas reducía significativamente la recurrencia de $36.4 \%$ con el cierre simple a $10 \%$ con malla en un periodo de seguimiento de un año. ${ }^{32}$

La desventaja principal del uso de mallas en el hiato es el riesgo de complicaciones locales (fibrosis y adherencias, erosión o perforación). ${ }^{30,33}$ No obstante, la incidencia de complicaciones relacionadas con el uso de mallas en el hiato es menor al $2 \%$, a pesar de que no se dispone de resultados a largo plazo (más de 10 años). En algunos casos, la erosión esofágica se ha reportado hasta más de dos años después de la cirugía. En ninguno de nuestros pacientes se ha presentado un caso de erosión esofágica, perforación o estenosis a pesar del uso de malla de polipropileno en los seis primeros casos, ya que ésta era la única que tenía la institución en ese momento; la literatura recomienda no utilizarlas por las complicaciones derivadas de su uso. Posteriormente, y hasta la fecha, utilizamos la malla bicapa, de la que se reporta menor índice de complicaciones relacionadas con su uso. En el último caso utilizamos una malla biológica, que a los dos años de seguimiento, no ha reportado complicaciones.

En un reciente metaanálisis de varias series no aleatorizadas de reparación laparoscópica de HHP en donde la inclusión estuvo restringida a aquellas series con más de 25 pacientes y un seguimiento mayor a seis meses, se estudiaron 965 pacientes, en quienes la recurrencia total fue del 10\%; sin embargo, en los grupos que eran evaluados con esofagograma postoperatoriamente, llegaba hasta el 25\%.34 Se apreció menor índice de recurrencia en quienes se realizó un alargamiento esofágico vía el procedimiento de gastroplastía de Collis Nissen versus aquéllos a quienes no se les realizó (0 versus $12 \%$ ). Nosotros no observamos acortamiento esofágico en ninguno de nuestros pacientes, por lo que no ha sido necesario realizar este tipo de procedimiento.

Reportes retrospectivos han mostrado que la morbimortalidad asociada con la reparación no electiva de la HHP ha sido significativamente mayor que después de una reparación electiva. ${ }^{35}$

Nuestro índice de mortalidad (0\%) es comparable al $0-3.7 \%$ reportado por Hashemi y Boushey. ${ }^{7,22}$ En nuestra población no hubo conversiones a cirugía abierta o complicaciones intraoperatorias. En el postoperatorio, dos pacientes tuvieron enfisema subcutáneo transitorio en el cuello, que se resolvió espontáneamente en menos de 24 horas.

El tratamiento de la hernia paraesofágica, en comparación con el resto de los pacientes con enfermedad por reflujo gastroesofágico, comprende un reto quirúrgico mayor debido a la presencia de una edad mayor que en las hernias tipo I. El riesgo de morbilidad y mortalidad postoperatorias es significativamente mayor en pacientes con hernia paraesofágica que en el resto de aquellos tratados quirúrgicamente por enfermedad por reflujo gastroesofágico, incluyendo eventos tromboembólicos, neumonía y laceraciones a otros órganos.

En nuestra serie hemos tenido tres pacientes que presentaron neumonía en el postoperatorio, tres atelectasias y un caso de neumotórax.

El análisis de las recidivas muestra diversos patrones en el momento y tipo de la presentación. Las hernias que ocurren inmediatamente en el postoperatorio, en general, son secundarias a la disrupción del cierre hiatal. Las observadas a largo plazo pueden adoptar varios patrones: HHP recurrente, la migración de la funduplicatura o una hernia pequeña por deslizamiento, sin una recidiva clara del saco paraesofágico. En el último subgrupo, la 
incidencia de síntomas es variable y muchas no se identifican si no se realiza un esofagograma.

La actitud terapéutica ante las recidivas sintomáticas es la reparación quirúrgica. Sin embargo, existe acuerdo tácito de que las recidivas no sintomáticas, en especial en el caso de pequeñas hernias por deslizamiento, deben ser simplemente controladas. ${ }^{33,34}$

Sabemos que dejar una porción del saco, sobre todo si tiene continuidad con el peritoneo, puede ser un camino posible de recidiva de la hernia. ${ }^{36}$ Esto es de suma importancia, aunque en ocasiones la profundidad mediastinal del saco y la idea fija de resecarlo pueden llevar a lesiones mortales (neumotórax, hemorragia, perforación esofágica) que dependen del tiempo de evolución y la fibrosis alrededor del saco herniario. ${ }^{37}$ El remanente peritoneal del saco en el tórax tiene poca importancia clínica; forma quistes de retención que son asintomáticos. ${ }^{38}$ En nuestra serie no fue posible resecar completamente el saco herniario en seis pacientes; sin embargo, se tuvo el cuidado de resecar los medios de fijación del mismo a los bordes del orificio herniario y la funduplicatura aplicada en todos los casos de nuestra serie fue la de Nissen; esto influye en la disminución del riesgo de recidiva. ${ }^{39}$

La reparación de la crura, también llamada "reparación crural" o "hiatoplastía", es uno de los pasos más importantes en la reparación de hernias hiatales. ${ }^{24}$ Cuschieri, en su primer reporte de reparación laparoscópica de HHP, cerró la crura con una sutura continua y fijó la funduplicación al margen anterior del hiato. ${ }^{19}$ Pronto se hizo evidente que el índice de recidiva de la HHP era inaceptablemente alto. Soricelli publicó una serie retrospectiva de 93 pacientes reparados con puntos separados de prolene, con seguimiento promedio de 95 meses y recurrencia de 19, con 91\% de satisfacción del paciente. ${ }^{26}$ Con esta técnica, algunas otras series han mencionado una recurrencia hasta del $40 \% .{ }^{33}$

La seguridad del uso de algunas prótesis es materia de preocupación debido a la disfagia a corto y largo plazo, producto de la fibrosis alrededor del esófago, además de los reportes de erosión o fistulización por cuerpo extraño. ${ }^{40-42}$

En cuanto a disfagia postoperatoria, en nuestra serie tenemos a 15 pacientes con re- sultados excelentes y buenos, tres con disfagia moderada y uno con disfagia leve. Lo anterior tal vez es influenciado por el hecho de tener el cuidado de permitir un centímetro libre entre la malla y el esófago.

Algunos autores han hecho énfasis en que la erosión intraluminal del esófago es la complicación más seria del uso de las prótesis y su prevalencia está reportada menor al 0.49\%. En una revisión de 1,446 pacientes, esta complicación —que eventualmente requeriría gastrectomía o esofagectomía - se presentó en el $0.07 \%{ }^{35}$

En teoría, las mallas sintéticas están asociadas a mayor riesgo de fibrosis, posible estenosis esofágica y, posteriormente, erosión esofágica o gástrica. ${ }^{43} \mathrm{~A}$ fin de minimizar estos efectos, además de la disfagia y la recidiva, se han desarrollado mallas biológicas de la dermis de cadáveres humanos y SIS (porcine small intestine submucosa). ${ }^{44}$ Éstas presentan una respuesta inflamatoria más leve y mejor neovascularización. Se cree que existe una reacción a cuerpo extraño limitada en el hiato debido a su biocompatibilidad, lo cual minimiza el riesgo de fibrosis y la consecuente disfagia postoperatoria. ${ }^{45}$

Un estudio a corto plazo que comparó el uso de sutura únicamente contra el de malla biológica, mostró que el índice de recurrencia varió de 16.6 a $3.5 \%$, respectivamente. ${ }^{45}$ En otro reporte sobre el uso de mallas biológicas SIS, el índice de recurrencia fue del $10 \%$ después de una mediana de seguimiento de 27 meses. El índice de recurrencia con la utilización de malla biológica SIS puede llegar hasta el $9 \%{ }^{46}$ En nuestra serie, el último caso que se incluyó fue intervenido con este tipo de malla biológica y hasta el momento no ha presentado disfagia ni recurrencia.

\section{CONCLUSIONES}

- A través del análisis de la evidencia podemos decir que la técnica ideal para el abordaje de las hernias paraesofágicas es la laparoscópica.

- Asimismo, cada vez existe mayor evidencia de que los materiales protésicos ayudan a disminuir la recurrencia de esta patología. 
- Es más seguro utilizar mallas dual mesh que de propileno.

- El lugar de las mallas biológicas está por demostrarse a través de los estudios actualmente en curso.

- A pesar de que hasta el momento no se han presentado complicaciones en esta serie con la utilización de malla de polipropileno, no se recomienda su uso, por la alta posibilidad de efectos adversos ya conocidos.

- La información disponible hasta ahora demuestra que el uso de mallas bicapa en la reparación de hernia hiatal es segura y previene la recidiva.

- En cuanto a las mallas biológicas, se requieren más estudios a largo plazo para valorar su efectividad.

\section{REFERENCIAS}

1. Hill LD, Kozarek RA, Kraemer SJ, Aye RW, Mercer CD, Low DE, et al. The gastroesophageal flap valve: in vitro and in vivo observations. Gastrointest Endosc. 1996; 44: 541-547.

2. Naunheim KS, Edwards M. Paraesophageal hiatal hernia. In: Shields TW, editor. General thoracic surgery. 7th ed. Philadelphia: Williams and Wilkins; 2009. pp. 1951-1960.

3. Haas $\mathrm{O}$, Rat $\mathrm{P}$, Christophe $\mathrm{M}$, Friedman $\mathrm{S}$, Favre JP. Surgical results of intrathoracic gastric volvulus complicating hiatal hernia. Br J Surg. 1990; 77: 13791381.

4. Dean C, Etienne D, Carpentier B, Gielecki J, Tubbs RS, Loukas M. Hiatal hernias. Surg Radiol Anat. 2012; 34: 291-299.

5. Violette A, Velanovich V. Quality of life convergence of laparoscopic and open anti-reflux surgery for gastroesophageal reflux disease. Dis Esophagus. 2007; 20: 416-419.

6. Navarro-Vargas LA, Ortiz de la Peña-Rodríguez JA, Orozco-Obregón P. Hernia hiatal, tratamiento quirúrgico actual; nuestra experiencia y revisión de la literatura. Rev Mex Cir Endoscop. 2012; 13: 20-23.

7. Hashemi M, Peters JH, DeMeester TR, Huprich JE, Quek M, Hagen JA, et al. Laparoscopic repair of large type III hiatal hernia: objective follow-up reveals high recurrence rate. J Am Coll Surg. 2000; 190: 553-560; discussion 560-561.

8. Luketich JD, Nason KS, Christie NA, Pennathur A, Jobe BA, Landreneau RJ, et al. Outcomes after a decade of laparoscopic giant paraesophageal hernia repair. J Thorac Cardiovasc Surg. 2010; 139: 395-404, 404.e1.

9. Goers TA, Cassera MA, Dunst CM, Swanström LL. Paraesophageal hernia repair with biomesh does not increase postoperative dysphagia. J Gastrointest Surg. 2011; 15: 1743-1749.

10. Paul MG, DeRosa RP, Petrucci PE, Palmer ML, Danovitch SH. Laparoscopic tension-free repair of large paraesophageal hernias. Surg Endosc. 1997; 11: 303-307.

11. Maziak DE, Todd TR, Pearson FG. Massive hiatus hernia: evaluation and surgical management. J Thorac Cardiovasc Surg. 1998; 115: 53-60; discussion 61-62.

12. Paul MG, DeRosa RP, Petrucci PE, Palmer ML, Danovitch SH. Laparoscopic tension-free repair of large paraesophageal hernias. Surg Endosc. 1997; 11: 303-307.

13. Marano L, Schettino M, Porfidia R, Grassia M, Petrillo M, Esposito G, et al. The laparoscopic hiatoplasty with antireflux surgery is a safe and effective procedure to repair giant hiatal hernia. BMC Surg. 2014; 14: 1.

14. Lundell LR, Dent J, Bennett JR, Blum AL, Armstrong D, Galmiche JP, et al. Endoscopic assessment of oesophagitis: clinical and functional correlates and further validation of the Los Angeles classification. Gut. 1999; 45: 172-180.

15. Wu JS, Dunnegan DL, Soper NJ. Clinical and radiologic assessment of laparoscopic paraesophageal hernia repair. Surg Endosc. 1998; 13: 497-502.

16. Mehta S, Boddy A, Rhodes M. Review of outcome after laparoscopic paraesophageal hiatal hernia repair. Surg Laparosc Endosc Percutan Tech. 2006; 16: 301-306.

17. Stylopoulos N, Gazelle GS, Rattner DW. Paraesophageal hernias: operation or observation? Ann Surg. 2002; 236: 492-500; discussion 500-501.

18. Ballian N, Luketich JD, Levy RM, Awais O, Winger D, Weksler B, et al. A clinical prediction rule for perioperative mortality and major morbidity after laparoscopic giant paraesophageal hernia repair. J Thorac Cardiovasc Surg. 2013; 145: 721-729.

19. Cuschieri A, Shimi S, Nathanson LK. Laparoscopic reduction, crural repair, and fundoplication of large hiatal hernia. Am J Surg. 1992; 163: 425-430.

20. Nguyen NT, Christie C, Masoomi H, Matin T, Laugenour K, Hohmann S. Utilization and outcomes of laparoscopic versus open paraesophageal hernia repair. Am Surg. 2011; 77: 1353-1357.

21. Stylopoulos N, Rattner DW. The history of hiatal hernia surgery: from Bowditch to laparoscopy. Ann Surg. 2005; 241: 185-193.

22. Boushey RP, Moloo H, Burpee S, Schlachta CM, Poulin EC, Haggar F, et al. Laparoscopic repair of paraesophageal hernias: a Canadian experience. Can J Surg. 2008; 51: 355-360.

23. Mori T, Nagao G, Sugiyama M. Paraesophageal hernia repair. Ann Thorac Cardiovasc Surg. 2012; 18: 297305.

24. Terry M, Smith CD, Branum GD, Galloway K, Waring JP, Hunter JG. Outcomes of laparoscopic fundoplication for gastroesophageal reflux disease and paraesophageal hernia. Surg Endosc. 2001; 15: 691-699.

25. Granderath FA, Schweiger UM, Kamolz T, Asche $\mathrm{KU}$, Pointner R. Laparoscopic Nissen fundoplication with prosthetic hiatal closure reduces postoperative intrathoracic wrap herniation: preliminary results of a prospective randomized functional and clinical study. Arch Surg. 2005; 140: 40-48.

26. Soricelli E, Basso N, Genco A, Cipriano M. Long-term results of hiatal hernia mesh repair and antireflux laparoscopic surgery. Surg Endosc. 2009; 23: 24992504. 
27. Tatum RP, Shalhub S, Oelschlager BK, Pellegrini CA. Complications of PTFE mesh at the diaphragmatic hiatus. J Gastrointest Surg. 2008; 12: 953-957.

28. Davis SS Jr. Current controversies in paraesophageal hernia repair. Surg Clin North Am. 2008; 88: 959-978, vi.

29. Cueto-García J. Uso de mallas en la plastía de hiato. Cir Gen. 2007; 29: 251-252.

30. Targarona EM, Balagué C, Garriga J, Trías M. Mallas en el hiato: Una controversia no solucionada. Rev Mex Cir End. 2004; 5: 44-57.

31. Zhang W, Tang W, Shan CX, Liu S, Jiang ZG, Jiang DZ, et al. Dual-sided composite mesh repair of hiatal hernia: our experience and a review of the Chinese literature. World J Gastroenterol. 2013; 19: 5528-5533.

32. Zou FS, Qin MF, Cai W, Zhao HZ. Laparoscopic mesh for massive esophageal hiatal hernia. Zhonghua Xiaohua Neijing Zazhi. 2010; 27: 636-638.

33. Targarona EM, Bendahan G, Balague C, Garriga J, Trias $M$. Mesh in the hiatus: a controversial issue. Arch Surg. 2004; 139: 1286-1296; discussion 1296.

34. Rathore MA, Andrabi SI, Bhatti MI, Najfi SM, McMurray A. Metaanalysis of recurrence after laparoscopic repair of paraesophageal hernia. JSLS. 2007; 11: 456-460.

35. Sihvo EI, Salo JA, Räsänen JV, Rantanen TK. Fatal complications of adult paraesophageal hernia: a population-based study. J Thorac Cardiovasc Surg. 2009; 137: 419-424.

36. Edye M, Salky B, Posner A, Fierer A. Sac excision is essential to adequate laparoscopic repair of paraesophageal hernia. Surg Endosc. 1998; 12: 1259-1263.

37. Floch NR, Hinder RA, Klingler PJ, Branton SA, Seelig $\mathrm{MH}$, Zuker KA. Surgical laparoscopy. 2nd edition. Philadelphia, PA: 2001. pp. 429-443.

38. Willekes $\mathrm{CL}$, Edoga JK, Frezza EE. Laparoscopic repair of paraesophageal hernia. Ann Surg. 1997; 225: 31-38.

39. Gupta A, Chang D, Steele KE, Schweitzer MA, Lyn-Sue J, Lidor AO. Looking beyond age and co-morbidities as predictors of outcomes in paraesophageal hernia repair. J Gastrointest Surg. 2008; 12: 2119-2124.

40. Tatum RP, Shalhub S, Oelschlager BK, Pellegrini CA. Complications of PTFE mesh at the diaphragmatic hiatus. J Gastrointest Surg. 2008; 12: 953-957.

41. Davis SS Jr. Current controversies in paraesophageal hernia repair. Surg Clin North Am. 2008; 88: 959-978, vi.

42. Antoniou SA, Koch OO, Antoniou GA, Pointner R, Granderath FA. Mesh-reinforced hiatal hernia repair: a review on the effect on postoperative dysphagia and recurrence. Langenbecks Arch Surg. 2012; 397: 19-27.

43. Antoniou SA, Koch OO, Antoniou GA, Pointner R, Granderath FA. Mesh-reinforced hiatal hernia repair: a review on the effect on postoperative dysphagia and recurrence. Langenbecks Arch Surg. 2012; 397: 19-27.

44. Kaleya RN. Evaluation of implant/host tissue interactions following intraperitoneal implantation of porcine dermal collagen prosthesis in the rat. Hernia. 2005; 9: 269-276.

45. Ward KC, Costello KP, Baalman S, Pierce RA, Deeken $\mathrm{CR}$, Frisella MM, et al. Effect of acellular human dermis buttress on laparoscopic hiatal hernia repair. Surg Endosc. 2015; 29: 2291-2297.

46. Antonakis F, Köckerling F, Kallinowski F. Functional results after repair of large hiatal hernia by use of a biologic mesh. Front Surg. 2016; 3: 16.

\section{Correspondencia:}

Dr. Jorge Fernando Ugalde Velásquez Hospital Regional $1 .^{\circ}$ de Octubre, ISSSTE. Av. Politécnico Nacional Núm. 1669, Col. Magdalena de las Salinas, 07760, Del. Gustavo A. Madero, Ciudad de México. E-mail: ugaldef@yahoo.com.mx 